\title{
Older people and the relationship between hospital services and intermediate care: results from a national evaluation
}

\section{Jon Glasby, Graham Martin and Emma Regen}

Jon Glasby, Senior Lecturer and head of health and social care, Health Services Management Centre, University of Birmingham

Graham Martin, Research Fellow, Institute for the Study of Genetics, Biorisks and Society, University of Nottingham

Emma Regen, Research Fellow, Leicester Nuffield Research Unit, Department of Health Sciences, University of Leicester

\section{Address for Correspondence}

Dr Jon Glasby

Health Services Management Centre

University of Birmingham

Park House, 40 Edgbaston Park Road

Edgbaston, Birmingham

B15 2RT

Tel

E-mail J.Glasby@bham.ac.uk

\section{Acknowledgements}

This paper is written in collaboration with the National Intermediate Care Evaluation team. This research was funded by the Department of Health and the Medical Research Council.

This manuscript has not been submitted to any other journal and there are no financial or other conflicts of interest. 


\title{
Older people and the relationship between hospital services
}

\section{and intermediate care: results from a national evaluation}

\begin{abstract}
Objectives: In the UK, new intermediate care services have been established to prevent unnecessary hospital admissions, facilitate effective discharge and prevent premature care home admissions. This paper reports findings from a national evaluation of intermediate care, focusing on the relationship between hospital services and intermediate care.
\end{abstract}

Methods: The evaluation was based on a survey of intermediate care co-ordinators, case studies with five 'whole systems' (Primary Care Trust areas) of intermediate care, and a systematic review of evidence for the effectiveness of intermediate care. This paper reports some findings from the case study work, drawing on interviews and focus groups with key managers and practitioners involved in the planning, management and delivery of intermediate care. Participants were asked about the development of intermediate care, the role and purpose of intermediate care, the organisation and management of intermediate care, strengths and weaknesses of intermediate care, relationships between intermediate care services and between intermediate care and 'mainstream' services, and future areas for development. The data were analysed using the framework approach. 
Results: Respondents identified a range of tensions between hospital services and intermediate care, including concerns about the role and involvement of acute clinicians; the safety, quality and appropriateness of intermediate care; access to and eligibility for intermediate care; a lack of understanding and awareness of intermediate care; and the risk of intermediate care being dominated by acute pressures.

Conclusions: Although participants were able to identify several practical ways forward, resolving such fundamental tensions seems to require significant and longterm cultural change in the relationship between acute and intermediate care. 


\section{Older people and the relationship between hospital services and intermediate care: results from a national evaluation}

\section{Background and Introduction}

Tackling delayed hospital discharge is currently a key government priority in the UK, with ministerial pledges to end widespread 'bed blocking' (1). To achieve this target, the Labour government has introduced a range of measures, including additional funding for health and social care and the introduction of new legislation (the Community Care (Delayed Discharges etc.) Act 2003) to charge social services departments for hospital beds unnecessarily 'blocked' by people awaiting social services provision (a system known as reimbursement). While hospital discharge has long been a problematic area of policy and practice (2), hospital admission has tended to receive less attention (until relatively recently). This is now beginning to change, however, with a growing focus on reducing emergency admissions and supporting people with long-term conditions (3). As a result of this, there are now national targets to reduce admissions, new case managers to maintain people with complex needs in the community, and a range of different admission avoidance schemes in place throughout the country.

For many commentators, the key to tackling delayed discharge and reducing emergency admissions is to increase the amount of rehabilitation and recuperation 
available, particularly for frail older people who tend to account for significant proportions of emergency admissions (4). As the Audit Commission suggests (5), older people's services often experience a 'vicious circle' (see figure 1). As the number of hospital admissions rises, lengths of hospital stays decline, opportunities for rehabilitation are reduced, there is an increased use of expensive residential/nursing home care and less money for preventative services, thereby leading to more hospital admissions.

\section{Take in figure 1}

Against this background, the development of intermediate care services has been promoted as one means of breaking out of the Audit Commission's 'vicious circle' and delivering on the government's commitment to joint working, prevention of unnecessary admissions and delayed discharges, and promoting independence. In 2000, The NHS Plan announced an extra $£ 900$ million to be invested over four years in intermediate care services such as rapid response teams, intensive rehabilitation services, recuperation facilities and integrated home care teams $(1,6)$. Although details were scarce at this stage, intermediate care was essentially designed to prevent unnecessary hospital admissions, facilitate swift and timely hospital discharges and prevent premature admissions to permanent residential and nursing care. While the term 'intermediate care' is a broad one that is used to refer to a wide range of diverse services (including rapid response, Hospital at Home, step-up and step-down care home places, supported discharge, and residential/day rehabilitation), the essential characteristics shared by such services were subsequently set out in a government 
circular (7), with intermediate care described as services that met all the criteria in figure 2 .

\section{Take in figure 2}

Whether the advent of intermediate care has the potential to break the Audit Commission's 'vicious circle' may very much depend on the relationship between existing acute hospital services and new intermediate care provision. Just as in the past there have been well documented tensions between community and acute care (2), there is clearly scope for similar tensions to emerge in intermediate care, and the ability of new services to act as a genuine alternative to and bridge out of hospital will be significant. Against this background, this paper reports findings from a national evaluation of intermediate care relating to the emerging relationship between acute and intermediate care services.

\section{Methods}

This paper reports findings from qualitative research undertaken by a team from the Universities of Birmingham and Leicester as part of a national evaluation of intermediate care. Commissioned by the Department of Health and the Medical Research Council, the evaluation lasted from January 2002 to June 2005. The research aims (set out in figure 3) were addressed via a range of research activities, including a questionnaire survey of intermediate care co-ordinators (Aims $1 \& 2$ ), case studies with 'whole systems' of intermediate care (Aims $3 \& 4$ ), and a systematic 
review of evidence for effectiveness of intermediate care (Aim 5). Five (Primary Care Trust-based) case study sites were included in the study, selected to include a mix of rural and urban areas where there has been a range of intermediate care services operational for at least 2-3 years, reasonable throughput into the intermediate care system (at least 1000 cases per annum), senior management support for the collection of routine data by services themselves, and clinical and managerial support for participation in the national evaluation.

\section{Take in figure 3}

Alongside quantitative data collection for economic analysis, in each area interviews $(n=61)$ and focus groups $(n=21)$ were held with key managers and practitioners involved in the planning, management and delivery of intermediate care. This included respondents from primary care, intermediate care, acute care and social services, together with focus groups with front-line staff. In both interviews and focus groups participants were asked about the background to the development of intermediate care services, the role and purpose of intermediate care, the organisation and management of intermediate care, strengths and weaknesses of intermediate care, relationships between intermediate care services (and between intermediate care and 'mainstream' services), and future areas for development. All interviews took place in private after written consent had been sought and interviewees had been given assurances of anonymity (data being presented in a non-attributable form). Interviews and focus-group discussions were tape-recorded and transcribed. The data were analysed using a method informed by the framework approach which involves 
the sifting, charting and sorting of material in a systematic process according to key issues and themes (8). As a result of this, key themes were identified from the data and subsequently discussed and validated at regular research team meetings. This paper focuses on data from interviews and focus groups, and explores data relating to the relationship between acute care and intermediate care.

\section{Findings}

Intermediate care as part of a spectrum of services and as a positive alternative to hospital

For many people, the development of intermediate care had initially been driven by the need to do things differently in order to reduce pressures on acute care. This was highlighted by a range of different respondents, including those from acute care, intermediate care and social care, and including both managers and clinicians. In particular, many respondents saw intermediate care as a positive concept with the potential to promote choice, independence and quality of life. For some, this contrasted significantly with the dependency culture which they felt existed in some acute settings, and could lead to older people returning to independent living in the community in a way in which stretched hospital services would not be able to achieve. In particular, the success of intermediate care was felt to hinge on the flexibility and choice it could offer as part of a wider spectrum of services for older people (see 9 for further discussion). 
In addition, a key feature of intermediate care was its capacity to invest time and resources in older people to help them regain their independence. Based outside hospital and in as homely an environment as possible, this was seen as preparing people to return home and as giving as realistic an insight as possible into people's self-care abilities in less institutional settings (see 10 for further discussion).

Difficulties in the relationship with acute care: issues for hospital staff

Despite these positive perceptions of intermediate care, some staff felt that local intermediate care services had been set up too quickly with insufficient input from hospital staff. For some, intermediate care represented a new project that took funding and focus away from existing good practice, and new services too often took the form of short-term, standalone projects, insufficiently linked to mainstream services:

"[Intermediate care] is still perceived as a bit of an add on and to a certain extent we still have a legacy of these projects and short termisms, and the difficulty with that is when you are working with the public or service users around intermediate care that kind of impacts on your working relationship because we don't want any more projects, we don't want anymore short term things, we want something that is going to be available on an ongoing basis." (site 5)

"I've been around far too long, I've seen so many new schemes come and go at the expense of good sound practice... [Sometimes it's not because existing schemes 
aren't working well, but because] the government likes to have new money going to new schemes and these new schemes [are] at the expense of [existing] good practice." (site 2 )

For some, these concerns were also linked to fears that intermediate care might represent a lower quality form of service for older people and that new models of service provision had been set up in advance of an appropriate evidence base. There were also concerns that clinicians in hospital settings may end up focusing solely on acute care and losing sight of the whole person, although some respondents felt that this constituted a more appropriate use of expensive acute capacity. For some clinicians, moreover, there was also a worrying trend towards avoiding the need for specialists - partly due to a desire to save money, but also through a desire to reduce medical power:

"I also feel there is a feeling amongst management in the sense that we must actually avoid specialists... and that means the care will be cheap. It is not, it may become more expensive. The specialists are there ... because... they can actually... get a better outcome for the patients, not for 'oh yes the control'. There are some doctors, they are not geriatricians themselves, a lot of them feel the old school were dictatorial, but modern doctors are not dictatorial, they want to work in a multidisciplinary [team], but if you actually want to avoid them and then develop pockets of services and other things it would be a disaster." (site 1)

In some services, moreover, there was a feeling that intermediate care was underused, with intermediate care services were regarded as somehow 'separate' or 'other' 
from mainstream provision. As a result, interviewees reported that many hospital staff (and indeed GPs too) lacked awareness and understanding of intermediate care services. Despite numerous attempts to promote intermediate care locally, interviewees reported that the concept had simply failed to become embedded within the mindsets of many mainstream practitioners. The rotation of hospital staff combined with pressures upon staff to find quick solutions were suggested as possible reasons for this:

"I just think people don't think about it naturally as it is fairly new. Services have been limited and where they are they are probably working at capacity because they are so limited so thinking of a route through intermediate care as an alternative to admitting somebody or discharge them into long-term care, people just don't think about it." (site 2)

In particular, key barriers included the gradual evolution of intermediate care (with different access points and eligibility criteria) and access criteria perceived by mainstream practitioners as being too restrictive. As a result, intermediate care could by seen by some as being rather detached from mainstream services, sometimes 'cherry-picking' patients. In addition, ongoing difficulties in making referrals to intermediate care could result in practitioners reverting to other forms of service provision:

"Well the units ... do develop criteria, don't they, because they have to safeguard themselves by having so many exclusions that actually they become almost impossible to use because busy clinicians can't maintain all the exclusion criteria at their 
fingertips. And if you refer and are rejected, refer and are rejected, next time you see a case you're going to think well, we'll do it as we always used to do." (site 1)

Difficulties in the relationship with acute care: issues for intermediate care staff

While there was general consensus about many of the issues for hospital staff set out above, some acute medical staff were also seen as resistant to change and as reluctant to refer to intermediate care for fear of losing control of their patients, and due to an ill-knowledge of what was available (which was not helped by frequent staff changes):

"I think the other thing is that I would like to see is that my colleagues in the hospital setting... feel more integrated with the intermediate care set up, which they don't at the moment... They don't understand what is out there and it is just so difficult to keep people up to speed with new developments and changes." (site 5)

"No I don't think safety is a problem, no. They just, I think these particular two [doctors] do not want to lose control of their patients. I think they see it as a threat, their patients going to somebody else, to a different Consultant." (site 1)

In addition, hospital services more generally were felt not to understand the nature of intermediate care, and there were concerns that intermediate care could become too dominated by a desire to reduce acute pressures: 
[We get inappropriate referrals, particularly when there's] a bed panic, like there is today, and everybody will be told to go through the ward and find any patients and there will almost be a blanket referral [to intermediate care] for virtually anybody who is vaguely upright." (site 1)

"I personally think we are perceived as someone that can empty a hospital bed and not as a continuation of the care." ( site 5)

Even where intermediate care reported a good relationship with the local hospital, comments from respondents sometimes suggested underlying misunderstandings of each other's role:

"We have built up a very good working relationship with [the] Hospital. I think the girls like the idea of having us at the back to polish up the bits they feel could be polished up but they haven't got the time to do." (site 4)

At the same time, there were also anecdotal and individual concerns that intermediate care beds in one site in particular were becoming 'blocked', with people discharged prematurely from hospital and no follow-up services to enable timely discharge from intermediate care. This was felt to be the result of a range of factors, including a lack of capacity in the home care and care home sector.

Often, intermediate care staff felt under pressure to accept as many referrals as possible, even if they suspected that some were inappropriate. As a new service, trying to win over the hearts and minds of staff in social, primary and acute care, 
some services felt that they had to be as helpful as possible to potential referrers and had to ensure that their beds were as full as possible to justify a need for their service:

"And one of the real hard things for Intermediate Care is that they are damned if they do and damned if they don't all the time, at the moment. So the minute we have no vacancies in the beds, people start jumping up and down. And then the minute we have vacancies in the beds they jump up and down for the opposite reason. If we can't accept somebody onto the team from the community they jump up and down. If we get it wrong and have a problem because we accept somebody we shouldn't have accepted, or whatever... And that at times is very hard for the people trying to deliver. Because they do feel shot at from all directions." (site 1)

"There is a pressure to use Intermediate Care services for things not fit for purpose. We are already being asked to put people in Intermediate Care places where there actually is not an Intermediate Care element to that. It is to get this person out of acute hospital bed." (site 2)

This task of winning over potential referrers was made more complex in one site as a result of the existence of two community hospitals, which tended to be much more regularly used by the local hospital than intermediate care. While some intermediate care services felt that they were sometimes seen as a means of relieving pressures in acute care, therefore, other services felt that they were under-utilised and were eager to receive more referrals: 
"We are not getting as many hospital discharges as we could have because of the close proximity to the two community hospitals, certainly in the past, it is easier for the staff on the acute hospital ward to just transfer straight across than to sort out how to make the access to Intermediate Care and set that up. We have probably not been utilised as much as we could have been and that still goes on." (site 3)

Overall, however, concerns from community staff about the dominance and practices of acute services were a recurring theme. As one person put it, "they are wary that they are a dumping ground for secondary care" (site 1). In response, participants suggested a number of ways forward that might help to ease tensions. In particular, respondents emphasised the need for greater geriatrician involvement in intermediate care to reassure hospital clinicians about the safety and quality of services; joint work to review eligibility criteria so that these are jointly owned and disseminated throughout both primary and acute care; and the creation of joint, rotational posts, with practitioners working in both intermediate care and acute settings. In addition, various sites emphasised the need for accessible information and publicity material to raise awareness, and for more proactive case-finding and in-reach into hospital (for example, with intermediate care taking full part in discharge meetings, diverting admissions from A\&E etc). However, underlying all these proposals was a sense that major cultural change will be required if hospital services and intermediate care are to work together more effectively in the future:

"The big cultural thing we found in particular about the intermediate care beds is hospital staff being prepared to take the risk and discharge somebody to something new that is relatively untested and unknown... So it is starting to overcome those 
barriers. Part of it is actually once somebody has put a patient through intermediate care then they have got the confidence to do it again." (site 4)

"I think the interface between primary and secondary care is a concept and it doesn't function really, other than as a place of passing people from one to the other by paper, or e-mail or whatever. I think our view is that you will only get a real interface if it's a working environment where there is some sort of working link between people in the community and people in hospital so that you can start to develop an understanding between clinicians of what is possible and so you can have some commonality about risk sharing and risk management..." (site 2)

\section{Discussion and Conclusions}

Part of a national evaluation of intermediate care, this paper has highlighted a number of significant tensions between acute and intermediate care. While participants from different professional and organisational backgrounds have different views, key issues include concerns about the role and involvement of acute clinicians in intermediate care; the safety, quality and appropriateness of intermediate care for some older people; access to and eligibility for intermediate care; a lack of understanding and awareness of intermediate care; and the risk of intermediate care being dominated by acute pressures. At times, this seems to be very much a case of opposites - some services feeling the need to respond to acute pressures while others feel underutilised; sometimes receiving inappropriate referrals but feeling the need to accept as many referrals as possible in order to embed the new service. 
Local context often accounts for these contrasts, for example the existence of alternative discharge arrangements which bypass intermediate care, as well as the differentiation between services and between sites. Variation in intermediate care provision within and between sites meant that some services were trusted partners of acute care clinicians, others were subject to overflows of inappropriate acute patients as they became 'holding bays' at times of pressure on hospital beds, while still others struggled to establish relationships with acute care and secure appropriate referrals in the first place. In part this was attributed to the effectiveness of information provision and communication, although it also seemed that hospital doctors were more willing to entrust the clinical safety of their patients to some services than others. This also serves as a reminder that many of the suggestions made by respondents above, about improving acute-intermediate care relationships, are likely to be fraught with political difficulties and clinical concerns, especially those which countenanced anything approaching a transfer of responsibility for hospital patients' safety.

While participants in the study had a range of practical suggestions as to how they might begin to the issues raised, many were long-term and depended on a series of relatively small-scale approaches that might in time begin to bring about the cultural change required to improve the relationship between intermediate care and hospital services. With a 2006 White Paper focusing on the need to develop 'care closer to home' (11) and with national targets to reduce emergency admissions, however, it is not clear whether intermediate care has this amount of time available to it (or indeed the power to tackle vested acute care interests). 
Perhaps in the long-run, the best way forward may be to focus more on providing the resources necessary to achieve swift and safe discharge and to focus on the whole system, not just on one part of it. Such an approach might well require additional funding for community services, possibly in conjunction with disinvestment in acute care. Above all, it would place greater emphasis on discharge practices in acute care. From existing literature, we know that some acute hospitals fail to plan ahead for discharge, do not adequately involve service users and their carers in decisions about discharge and aftercare, and do not give sufficient notice to users/carers and other services $(2,12-16)$. Viewed from this perspective, finding the best way of facilitating positive relationships between acute services and intermediate care may well be a crucial aspect of current attempts to tackle delayed discharge and rising emergency admissions.

Overall, this insight into the relationship between hospital discharge and intermediate care raises significant concerns about the extent to which new intermediate care services - so widely heralded in 2000 - have the capacity to rebalance the current health and social care system and make significant inroads into current emergency admission and delayed discharge rates. Whilst intermediate care may remain a positive step forward with considerable potential, there is a danger that this potential may not be fully realised unless some of the tensions and barriers identified in this paper are resolved. 


\section{References}

1. Department of Health. The NHS plan: a plan for investment, a plan for reform. London: The Stationery Office (TSO), 2000

2. Glasby J. Hospital discharge: integrating health and social care. Abingdon: Radcliffe Medical Press, 2003

3. Department of Health. The NHS improvement plan: putting people at the heart of public services. London: Department of Health, 2004

4. Blatchford O, Capewell S, Murray S, Blatchford M. Emergency medical admissions in Glasgow: general practices vary despite adjustment for age, sex, and deprivation. British Journal of General Practice 1999; 49: 551-554

5. Audit Commission. The way to go home: rehabilitation and remedial services for older people. London: Audit Commission, 2000

6. Department of Health. National service framework for older people. London: TSO, 2001

7. Department of Health. Intermediate care. Circular HSC 2001/01, LAC(2001)1, 2001 
8. Ritchie J, Spencer L. Qualitative analysis for applied policy research. In A. Bryman and R.G. Burgess (eds) Analysing qualitative data. London: Routledge, 1994

9. Regen E, Martin G, Glasby J, Hewitt G, Nancarrow S, Parker H. A national evaluation of intermediate care (1): challenges, benefits and weaknesses - qualitative study. Unpublished paper submitted to British Medical Journal, 2006

10. Martin GP, Nancarrow SA, Parker H, Phelps K, Regen EL. Place, policy and practitioners: on rehabilitation, independence and the therapeutic landscape in the changing geography of care provision to older people in the UK. Social Science \& Medicine 2005; 61: 1893-1904

11. Department of Health. Our health, our care, our say. London: Department of Health, 2006

12. Henwood M, Hardy B, Hudson B, Wistow G. Inter-agency collaboration: hospital discharge and continuing care sub-study. Leeds: Nuffield Institute for Health Community Care Division, 1997

13. Horne D. Getting Better? Inspection of hospital discharge (care management) arrangements for older people. London: Department of Health, 1998

14. House of Commons Health Committee. Delayed discharges (third report). London: TSO, 2002 
15. Neill J, Williams J. Leaving hospital: older people and their discharge to community care. London: HMSO, 1992

16. Tierney A, Worth A, Closs SJ, King C, Macmillan M. Older patients' experiences of discharge from hospital. Nursing Times, 1994. 25(90): 36-39 
Figure 1 The 'Vicious Circle'

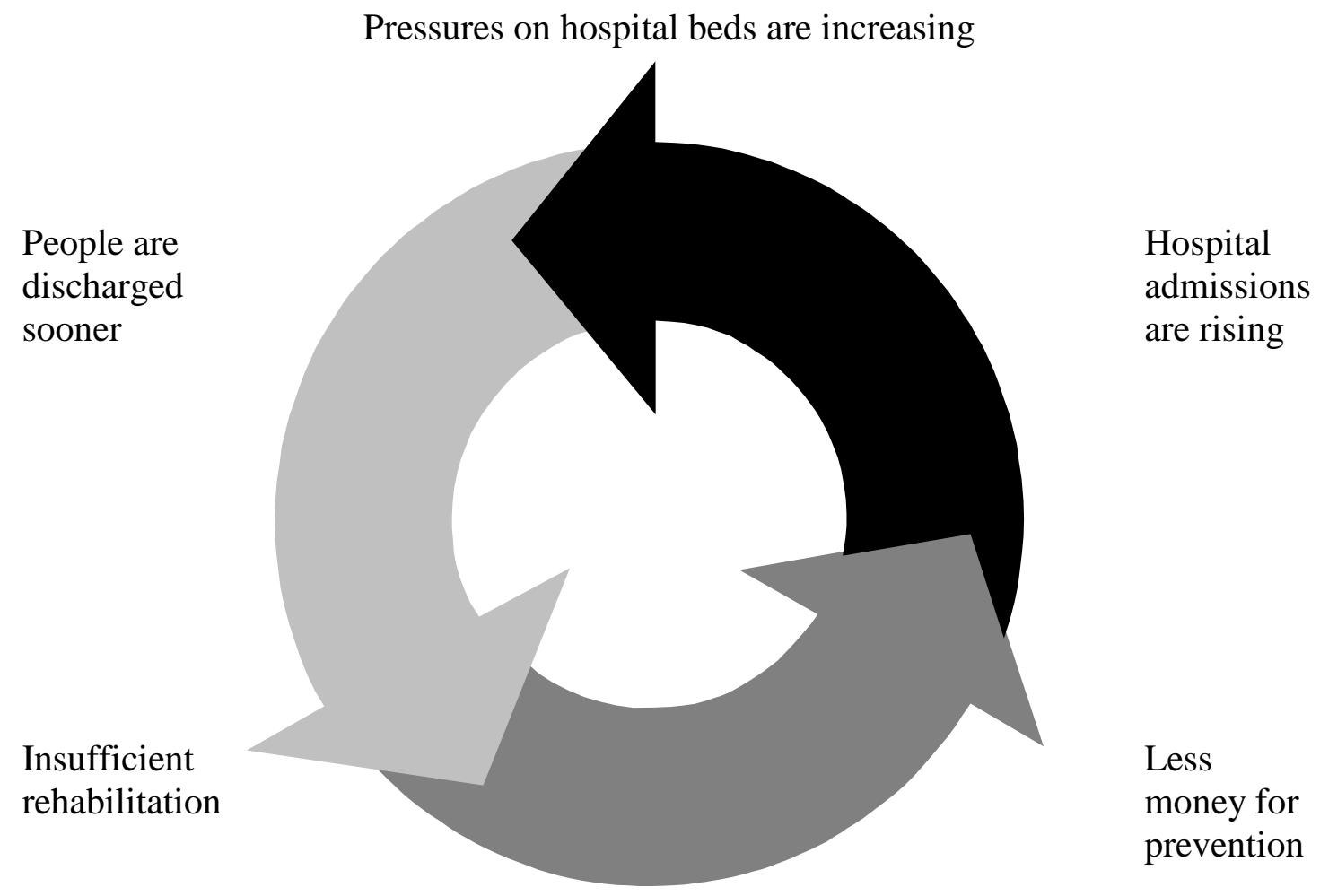

Increasing use of expensive

residential/nursing care

Source: Adapted from the Audit Commission (5) 
Figure 2: Intermediate care services

- Are targeted at people who would otherwise face unnecessarily prolonged hospital stays or inappropriate admission to acute inpatient care, long-term residential care, or continuing NHS inpatient care.

- Are provided on the basis of a comprehensive assessment, resulting in a structured individual care plan that involves active therapy, treatment or opportunity for recovery.

- Have a planned outcome of maximising independence and typically enabling patients/users to resume living at home.

- Are time-limited, normally no longer than six weeks and frequently as little as 1-2 weeks or less.

- Involve cross-professional working, with a single assessment framework, single professional records and shared protocols. 


\section{Figure 3: Research Aims}

1. To establish the range and extent of intermediate care service provision across England.

2 To explore the views of intermediate care leads on the benefits and challenges of implementing intermediate care policy.

3 To assess the impact of intermediate care on the service system as a whole and on individual service users.

4 To explore the costs of intermediate care schemes in relation to their outcomes.

5 To synthesise evidence from this and other research on the costs and outcomes of different models of intermediate care and on best practice. 